\section{Uso de la toxina botulínica en el tratamiento de sonrisa gingival}

\section{Use of botulinum toxin in the treatment of gummy smile}

\section{Resumen}

El objetivo es abordar la importancia del uso de la toxina botulínica para corregir la sonrisa gingival, demostrando la satisfacción del paciente, aunque el efecto sea temporal y reversible, evitando procedimientos invasivos. Se realizó un levantamiento bibliográfico en las bases de datos PubMed, LILACS y Bireme, los términos utilizados en la investigación fueron: sonrisa gingival y toxina botulínica o sonrisa gingival y estética. Los criterios de inclusión fueron una revisión de literatura de casos clínicos de pacientes con sonrisa gingival publicados entre 2015 y mayo de 2019. Esta revisión aborda la aplicación de la toxina botulínica para devolver una sonrisa armoniosa y hermosa al paciente, ya que es una alternativa viable, eficiente, menos invasiva y segura. La práctica de la aplicación de toxina botulínica puede ser un complemento útil para mejorar la estética y mejorar la satisfacción del paciente en relación a la sonrisa gingival sin realizar procedimientos invasivos, es decir, cuando está debidamente indicado, pudiendo reemplazar o agregar procedimientos quirúrgicos.

Palabras clave: Toxinas botulínicas tipo A; Sonrisa; Odontología; Estética (fuente: DeCS BIREME).

\begin{abstract}
The objective was to address the importance of using botulinum toxin to correct gummy smile, showing patient satisfaction even if the effect is temporary and reversible, avoiding invasive procedures. A bibliographic survey was carried out in the databases PubMed, LILACS and Bireme, the terms used in the research were gummy smile and botulinum toxin or gummy smile and aesthetic. The inclusion criteria were a review of the literature of clinical cases of patients with with a gummy smile published between 2015 and May 2019. This review addressed the application of botulinum toxin to give the patient a beautiful and harmonious smile, as it is a viable, efficient, less invasive and safe alternative. The practice of applying botulinum toxin can be a useful complement to improve patient satisfaction with a gummy smile without undergoing invasive procedures, that is, when well indicated, being able to replace or complement surgical procedures.
\end{abstract}

Keywords: Botulinum toxins; Smiling; Dentistry; Aesthetics (source: MeSH NLM).

\section{Artículo de Revisión}

Mirilaini Lino Brancini ${ }^{1, a}$, Danielle Cristina Alves Teodoro ${ }^{1, a}$, Taylane Soffener Berlanga De Araújo ${ }^{1,2, b}$, Andréa Candido dos Reis $3, b$

${ }^{1}$ Centro Universitário do Norte Paulista (UNORP), Universidade de Odontologia, São José do Rio Preto, São Paulo, Brasil

${ }^{2}$ Unidades Integradas De Pós-Graduacão, Pesquisa e Extensão (Unipós), São José do Rio Preto, São Paulo, Brasil.

${ }^{3}$ Universidade de São Paulo (USP), Faculdade de Odontologia de Ribeirão Preto, Ribeirão Preto, São

Paulo, Brasil

a Estudiante de Odontología.

b Doctora en Odontología.

\section{Correspondencia:}

Taylane Soffener Berlanga De Araújo: tsoffener3@gmail.com Rua Márcio Antônio De Pádua Guimarães, 94 CHF 12. Recreio Itanhangá, Ribeirão Preto. São Paulo-Brasil, CEP: 14.094-550.

ORCID: 0000-0003-1879-7727

\section{Coautores:}

Mirilaini Lino Brancini: mirilaini@outlook.com

ORCID: 0000-0002-8783-7131

Danielle Cristina Alves Teodoro: danieleteodoro01@ gmail.com

ORCID: 0000-0002-1714-1470

Andréa Candido dos Reis: andreare73@yahoo.com.br ORCID: 0000-0002-2307-1720

\section{Editora:}

Sandra Patricia Palomino-Gómez

Universidad Nacional Mayor de San Marcos, Perú.

Conflicto de intereses: los autores declaran no tener conflictos de interés.

Fuente de financiamiento: autofinanciado.

Recibido: 23/09/20

Aceptado: 22/02/21

Publicado: 01/04/21

(c) Los autores. Este artículo es publicado por la revista Odontología Sanmarquina de la Facultad de Odontología, Universidad Nacional Mayor de San Marcos. Este es un artículo de acceso abierto, distribuido bajo los términos de la licencia Creative Commons Atribución 4.0 Internacional (CC BY 4.0) [https://creativecommons.org/ licenses/by/4.0/deed.es] que permite el uso, distribución y reproducción en cualquier medio, siempre que la obra original sea debidamente citada de su fuente original. 


\section{Introducción}

La sonrisa de un paciente puede imprimir la sensación de alegría, éxito, sensualidad, afecto y cortesía, donde una sonrisa es más que una forma de comunicación, es el medio de socialización y atracción. En vista de ello, la Odontología es un gran aliado, no solo por el restablecimiento de la función y bienestar, sino principalmente por la búsqueda de una sonrisa en armonía con una cara equilibrada, lo es que es definido como belleza y juventud ${ }^{1,2}$.

De esta forma, la exposición de la gingiva en exceso en la sonrisa puede llevar a la insatisfacción en algunos pacientes y no siempre la opción quirúrgica es aceptada, ya que los procedimientos quirúrgicos demandan tiempo y riesgos, los cuales pueden provocar recelo, miedo e incluso desistir del tratamiento ${ }^{3}$.

Matos et al. ${ }^{3}$ explican que existen algunas clasificaciones para la sonrisa gingival envolviendo grupos musculares diferentes: en la sonrisa gingival anterior los principales músculos involucrados son los elevadores del labio superior y del ala nasal, y los principales músculos involucrados en la sonrisa gingival posterior son los zigomáticos mayores y los zigomáticos menores, mientras que en la sonrisa asimétrica se debe hacer una evaluación para cada caso con el fin de identificar los músculos involucrados en el lado de mayor tonicidad muscular.

Una sonrisa armónica debe ser simétrica y tener una exposición menor a 2 milímetros de gingiva, cualquier exposición gingival durante la sonrisa por encima de esa medida es conocida como sonrisa gingival ${ }^{4-6}$.

Los factores etiológicos de la sonrisa gingival son: hiperplasia gingival, protrusión dento-alveolar superior, exceso vertical maxilar (VME), extrusión dento-alveolar anterior y/o erupción pasiva alterada, hiperactividad de los músculos del labio superior durante la sonrisa o labios superiores cortos, hipertrofia gingival infecciosa $y / o$ medicamentosa, y características morfofuncionales del labio superior ${ }^{7}$.

Siendo las opciones de tratamiento de sonrisa gingival no quirúrgico - aplicación de toxina botulínica y el tratamiento ortodóntico; cirugías - osteotomía Le Fort I, resección ósea, gingivectomía, reposición labial, manipulación quirúrgica de músculos labiales específicos y microimplantes ${ }^{8,9}$.

Una vez establecido el diagnóstico de la sonrisa gingival, una modalidad de tratamiento mínimamente invasiva, que puede servir como coadyuvante o sustituto para el procedimiento quirúrgico es el uso de toxina botulínica. La toxina botulínica puede ser una terapia alternativa más rápida para tratar la sonrisa gingival ${ }^{10}$.

Así, esta revisión de literatura tiene como objetivo abordar la importancia del uso de la toxina botulínica en la corrección de la sonrisa gingival, mostrando la satisfacción del paciente, aunque el efecto sea temporal y reversible evitando procedimientos invasivos.

\section{Métodos}

Las bases de datos PubMed, LILACS y Bireme sirvieron para buscar los términos sonrisa gingival, toxina botulínica y estética. Los criterios de inclusión fueron revisiones de literatura de casos clínicos de pacientes que presentaban sonrisa gingival, publicados en el periodo de 2015 hasta mayo 2019.

\section{Tratamiento para la sonrisa gingival}

El primer paso para establecer un diagnóstico correcto de sonrisa armónica es clasificar adecuadamente el nivel gingival, respetando las variables como género, edad y salud periodontal; una vez determinada la anormalidad del nivel de la sonrisa, el establecimiento de su etiología es imprescindible ${ }^{1}$.

Chagas et al. ${ }^{11} \mathrm{y}$ Pedron et al. ${ }^{12}$ afirman que existen varios procedimientos que pueden ser realizados para la corrección de la sonrisa gingival como: gingivoplastía, intrusión de dientes por medio de aparato ortodóntico, cirugía ortognática y resección ósea, los cuales están disponibles para reducir la sonrisa gingival. No obstante, esos tratamientos son altamente complejos y costosos. Además explican que la exposición gingival excesiva causada por un labio superior hiperactivo puede ser corregido usando reposición labial quirúrgica, miotomias, desprendimiento del musculo labia y terapia con toxina botulínica tipo A.

La toxina botulínica A ha sido ampliamente utilizada en Odontología para el tratamiento de la hiperfunción muscular como es el caso de la sonrisa gingival, además de desórdenes temporomandibulares, asimetrías faciales, hipertrofia masetérica, espasmo hemifacial, dolor miofacial, sialorrea y bruxismo ${ }^{1,3}$.

Matos et al. ${ }^{3}$ explican que cada local de aplicación posee dosis de acuerdo con las características y necesidades individuales, incluyendo la tonicidad de los músculos involucrados.

La sonrisa es controlada por varios músculos faciales, como el elevador del labio superior y el ala de la nariz, zigomático menor y mayor, elevador del ángulo de la boca, risorio y orbicular de la boca; cada músculo involucrado en la elevación del labio superior tiene una función durante la actividad de sonreír ${ }^{12}$ (Figura y Tabla ).

La figura proporciona la visualización de los músculos responsable de la sonrisa de las personas. Cada musculo responsable por la elevación del labio superior, consecuentemente, la sonrisa, presenta una función peculiar durante el curso de esta expresión facial de alegría, por ello, es necesario conocer la clasificación de la sonrisa gingival y qué músculos son de la toxina botulínica, así como el método de aplicación de la toxina botulínica adecuado para cada caso ${ }^{13}$.

\section{Etiología de la sonrisa gingival}

Según Chagas et al. ${ }^{11}$ la etiología de la sonrisa gingival puede implicar una actividad labial (contracción muscular excesiva), longitud del labio, longitud de la coro- 


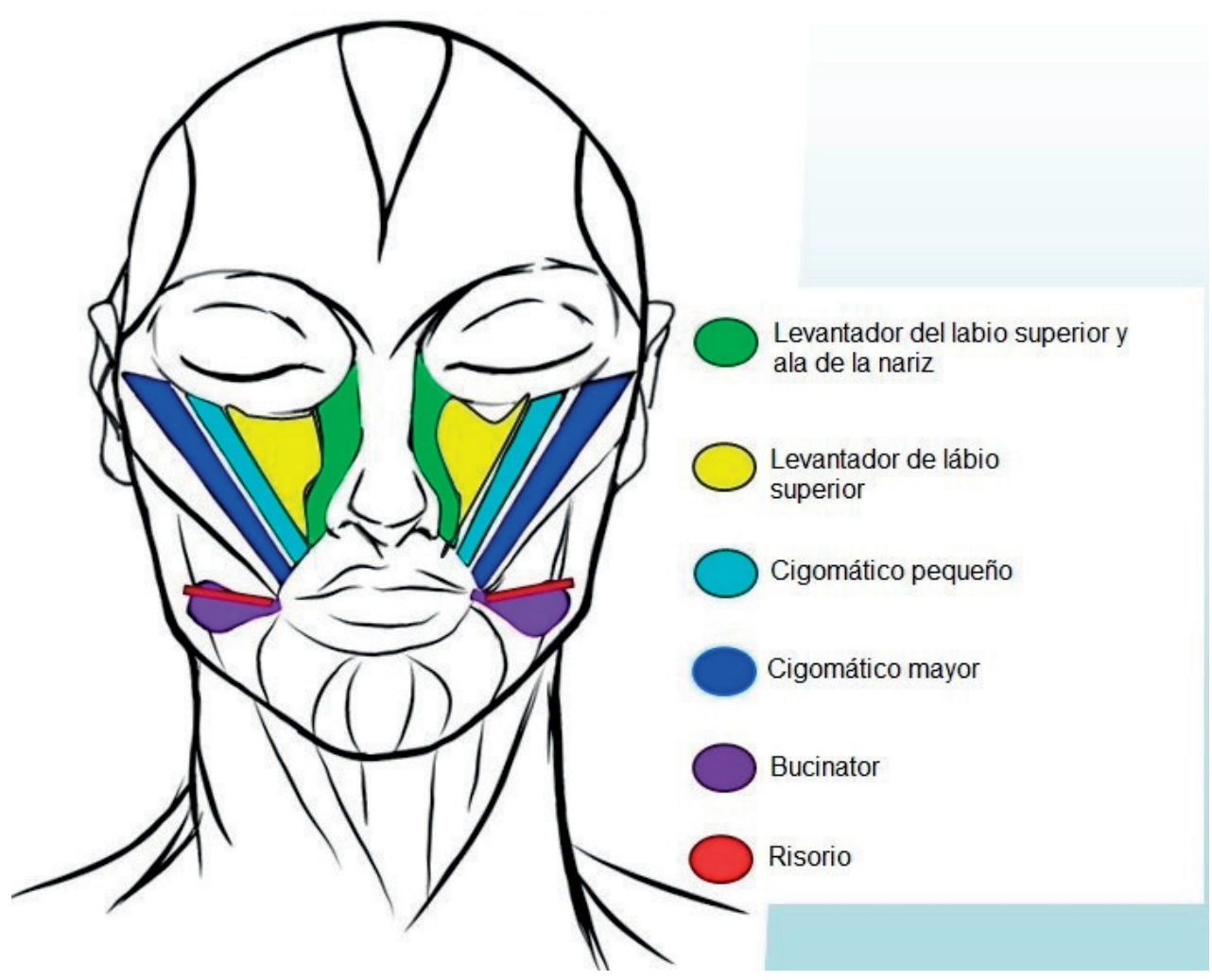

Figura. Músculos de la sonrisa

Fuente: Dibujo basado en el libro de Madeira, 2001.

Tabla. Regiones anatómicas para aplicación de la toxina botulínica en la corrección de la sonrisa gingival

\begin{tabular}{ll}
\hline \multicolumn{1}{c}{ Tipos de Sonrisa } & \multicolumn{1}{c}{ Músculos involucrados } \\
\hline Sonrisa gingival anterior & Elevador del labio superior y ala de la nariz y levantador del labio superior \\
Sonrisa gingival posterior & Cigomático mayor y menor \\
Sonrisa gingival mixta & Elevador del labio superior y ala nasal, levantador del labio superior y cigomático mayor y menor \\
Sonrisa gingival asimétrica & Elevador del labio superior y/o cigomático mayor o menor, en el lado donde hay exposición gingival) \\
\hline
\end{tabular}

Fuente: Matos et al., 2017.

na clínica, extrusión alveolar, extrusión dentoalveolar y exceso vertical superior. Matos et al. ${ }^{3}$ completan que la etiología de la sonrisa gingival está asociada a diversos factores: protrusión dentoalveolar superior, exceso vertical maxilar, hiperfunción muscular de los músculos elevadores del labio superior y extrusión o erupción pasiva alterada de los dientes anterosuperiores.

El cenit gingival es el punto más apical del contorno gingival y en los dientes superiores está localizado distalmente con el eje dental; en la sonrisa ideal el contorno del margen gingival debe ser paralelo a la curva incisal y a la curvatura del labio inferior ${ }^{3}$.

Varias etiologías han sido sugeridas para la sonrisa gingival, como el exceso vertical, retraso en la erupción pasiva, hiperfunción de los músculos involucrados en la sonrisa y la longitud reducida de la corona clínica de los dientes, que pueden producirse separadamente o en conjunto y determinar el tipo de tratamiento a utilizar ${ }^{14}$.
Kuhn-Dall'Magro et al. ${ }^{1}$ informaron que, al sonreír, el labio superior se desplaza apicalmente, dejando al descubierto los dientes anteriores y los márgenes gingivales; en esta situación, normalmente, se ponen de manifiesto de 1 a $2 \mathrm{~mm}$ de encía.

La sonrisa está formada por la unión de tres componentes: diente, encía y labios, que deben estar dispuestos en una proporción adecuada, y cuando esa proporción se excede, sugiere una condición no estética conocida como sonrisa gingival ${ }^{12}$.

El diagnóstico clínico de la sonrisa gingival debe incluir la determinación de la longitud clínica de la corona (margen gingival al borde incisal), la longitud anatómica de la corona (unión cemento-esmalte al borde incisal), la profundidad de sondaje (margen gingival a la base del surco gingival), la longitud de la encía queratinizada (margen gingival libre a la unión mucogingival), 
la fijación del frenillo, el espacio de overjet y overbite de los dientes límites verticales de la sonrisa ${ }^{15}$.

Kuhn-Dall'Magro et al. ${ }^{1}$ explicaron que se han propuesto varios factores etiológicos, que pueden dividirse en: dentarios, gingivales, óseos y muscular.

Matos et al. ${ }^{3}$ aclararon que, para elegir las diferentes opciones de tratamiento para la sonrisa gingival, se deben tener en cuenta algunos criterios y parámetros, como la exposición dental en reposo, la exposición dental durante la sonrisa, la posición del borde incisal en relación con el labio inferior, las pruebas fonéticas, el tamaño y la proporción dental, la prevención o el restablecimiento de la guía anterior, la forma y la longitud de la raíz y el soporte periodontal.

\section{Aplicación, dosis y durabilidad de la toxina botulínica}

La preparación de la toxina es específica para cada marca y el cirujano dental debe leer detenidamente las instrucciones de uso para saber exactamente la proporción. El producto se vende en un pequeño frasco que contiene $50 \mathrm{U}, 100 \mathrm{U}$ o $200 \mathrm{U}$ (unidades) de toxina, en forma de polvo liofilizado, estéril y envasado al vacío. Debido al vacío, se debe tener cuidado durante la dilución, evitando que la introducción de la solución salina se produzca rápidamente, provocando la agitación del fármaco, lo que podría alterar sus moléculas ${ }^{10}$.

Chagas et al. ${ }^{11}$ sugieren que la dosis y los sitios de aplicación deben ser personalizados de acuerdo con la gravedad del caso, y además afirma que la reducción de la exposición gingival puede variar de 3,04 $\mathrm{mm}$ a $5,11 \mathrm{~mm}$ después de 2 semanas de aplicación. Y coinciden en que la toxina botulínica debe administrarse con precaución, porque muchos factores externos y las características individuales de los pacientes pueden influir en los efectos de su aplicación. Factores como la inyección adecuada de la toxina en el músculo, la concentración de la solución, la susceptibilidad individual y la variación metabólica pueden influir en la longevidad de los efectos de la toxina botulínica.

En la sonrisa gingival se utiliza como máximo un total de $20 \mathrm{U}$, donde 2 a $4 \mathrm{U}$ son dividas por los lados a aplicar. El paciente debe ser orientado sobre los cuidados posteriores a la aplicación de la toxina botulínica, evitando masajear la región tratada poco después de la aplicación, manteniendo una posición erguida y no acostándose durante las primeras 4 horas después de la aplicación, y evitando el ejercicio físico durante las primeras 24 horas después de la aplicación ${ }^{3}$.

Kuhn-Dall'Magro et al. ${ }^{1}$ afirmaron que el resultado de la aplicación tenderá a cambiar debido al mecanismo farmacológico de la toxina botulínica $A$, y que en un periodo medio de 3 a 6 meses el paciente necesitará una nueva aplicación del producto.

Mostafa ${ }^{15}$ concuerda que el efecto de la toxina botulínica se observa en el plazo de 1 a 2 semanas y suele durar de 4 a 6 meses. Además, Pedron et al. ${ }^{10}$ también concuerda y afirma que los efectos clínicos aparecen en 2-10 días después de la inyección y el efecto más visible se produce 14 días después de la inyección, este primer efecto programado para ser progresivo también es reversible y dura alrededor de 3 a 6 meses.

Los profesionales deben saber cómo administrar la dosis, la técnica y la localización de la punción para evitar complicaciones ${ }^{16}$. Pedron et al. ${ }^{10}$ explican que la toxina botulínica cuando se inyecta puede extenderse concéntricamente en un área de 1 a $30 \mathrm{~mm}$, lo que permite una extensión efectiva.

\section{Toxina botulínica}

La toxina botulínica es una proteína producida por el Clostridium botullinum, una bacteria anaerobia Gram-positiva que causa el botulismo. Existen siete serotipos de toxinas (A, B, C1, D, E F y G), sin embargo, la toxina de tipo A es la más utilizada, porque es más eficiente y tiene menos costo ${ }^{10,12,14}$.

Chagas et al. ${ }^{11}$ coinciden en que existen varios subtipos de toxina botulínica, y la toxina botulínica A fue aprobada para uso cosmético debido a su seguridad y eficacia clínica, mostrando su eficacia en la corrección de la sonrisa gingival debido a su aplicación directa y segura en pequeñas dosis, rápida acción inicial, bajo riesgo y efectos reversibles.

Kuhn-Dall'Magro et al. ${ }^{1}$, Cavalcanti et al. ${ }^{2}$, Matos et al. ${ }^{3}$ y Chagas et al. ${ }^{11}$ relataron de que la toxina botulínica inhibe la liberación de acetilcolina, un neurotransmisor responsable de activar la contracción muscular y la secreción de las glándulas, reduciendo el tono muscular en el lugar de aplicación.

La dilución de la toxina botulínica tipo A se produce mediante una inyección suave de diluyente inyectable (cloruro de sodio al $0,9 \%$ ); y la solución debe almacenarse entre 2 y $8^{\circ} \mathrm{C}^{14,17}$.

La reversión de la parálisis local promovida por la toxina botulínica se produce por dos mecanismos: por germinación neuronal, donde se produce la formación de brotes axonales, la reinervación; y la formación de nuevas placas terminales más pequeñas con reinervación muscular por regeneración de las proteínas de acoplamiento de las vesículas de acetilcolina cuya función suele restablecerse entre tres y cuatro meses ${ }^{3}$.

\section{Odontología mínimamente invasiva}

Actualmente, la Odontología, además de hacer hincapié en la promoción de la salud, también busca la excelencia estética de la sonrisa, ya que es una forma de comunicación y socialización que expresa varios sentimientos ${ }^{12}$.

Kuhn-Dall'Magro et al. ${ }^{1}$ explicaron que, desde un punto de vista anatómico y fisiológico, la sonrisa es el resultado de la exposición de los dientes y las encías durante la contracción de los grupos musculares de los tercios medio e inferior de la cara.

En una sonrisa agradable, el borde del labio superior debe organizarse simétricamente hasta $3 \mathrm{~mm}$ de la en- 
cía, y la línea de la encía debe seguir el contorno de la exposición del labio superior; de esa forma exhibir más de $3 \mathrm{~mm}$ de encía en la sonrisa es llamada sonrisa gingival, la cual es considerada un disturbio estético ${ }^{11,16}$.

Según Matos et al. ${ }^{3}$, dependiendo del diagnóstico, el tratamiento para la corrección de la sonrisa gingival puede implicar la terapia periodontal, la cirugía ortognática, el tratamiento de ortodoncia o el uso de la toxina botulínica tipo A asociada o no a una de esas terapias.

Matos et al. ${ }^{3}$ informaron de que la complejidad de algunas cirugías suele llevar a los pacientes a renunciar a los planes de tratamiento que implican procedimientos más invasivos. Hay una concordancia entre los autores en que a veces el procedimiento quirúrgico, además de ser más invasivo, no favorece el resultado esperado por el paciente, siendo la toxina botulínica otra alternativa segura, rápida y eficaz para la corrección de la sonrisa gingival, siempre que se respete la dosis y el tipo de sonrisa ${ }^{12}$.

\section{Riesgos y complicaciones durante la aplicación de la toxina botulínica}

Chagas et al. ${ }^{11}$ destacaron que varias aplicaciones de toxina botulínica pueden conducir a una disminución permanente de la capacidad de contracción muscular, aunque los efectos de la toxina desaparezcan.

Matos et al. ${ }^{3}$ y Moreira et al. ${ }^{10}$ advirtieron que el uso de la toxina botulínica se considera un procedimiento seguro y eficaz, sin embargo, puede asociarse a posibles complicaciones como reacción alérgica, eritema, dolor, edema en el lugar de la inyección, adormecimiento temporal, náuseas, dolor de cabeza, hipoestesia transitoria, parálisis no deseada de los músculos adyacentes, alteración de la voz y xerostomía. De acuerdo con ello, Pedron et al. ${ }^{12}$ agregaron que, a pesar de ser un procedimiento seguro, la aplicación de toxina botulínica puede desencadenar eventos adversos como hematoma, dolor local, infección, edema, disfonía, disfagia, elongación del labio superior y asimetría de la sonrisa.

Mostafa ${ }^{15}$ confirma que los efectos secundarios incluyen dolor, infección, hematoma, inflamación, edema, pérdida de fuerza muscular, parálisis nerviosa y hematoma.

Los autores Moreira et al. ${ }^{10}$ y Mostafa ${ }^{15}$ coincidieron en que las contraindicaciones para el uso de la toxina botulínica son para los pacientes que presentan miastenia gravis y síndrome de Lambert Eaton y otras enfermedades degenerativas neuromusculares, las mujeres lactantes o embarazadas, los individuos con hipersensibilidad a uno de los componentes de la fórmula y los pacientes que están utilizando antibióticos clasificados como aminoglucósidos.

Matos et al. ${ }^{3}$ y Pedron et al. ${ }^{12}$ afirmaron que el uso de la toxina botulínica también está contraindicado en casos de pacientes con procesos inflamatorios presentes en la piel y en el sitio donde se realiza la aplicación, alergia a la albúmina u otros agentes que interfieren en la transmisión neuromuscular, y pacientes con enfermedades en el sistema nervioso periférico o con trastorno neuromuscular.

Además, una técnica de inyección inadecuada puede dar lugar a un aspecto asimétrico de la sonrisa, a algunas dificultades para hablar, masticar y/o beber, donde una administración excesiva puede provocar la caída del labio o la ptosis por debajo del margen gingival, causando la obstrucción de los dientes visibles en una sonrisa completa ${ }^{15}$.

Cuando se produce una sobredosificación se observan reacciones adversas como disfagia y disartria y dificultades en la masticación, o incluso cuando la infiltración no alcanza el músculo objetivo cubriendo las estructuras adyacentes al músculo. Los síntomas como el dolor de cabeza, el letargo y el dolor muscular sólo se producen como resultado de una dosis excesiva, pero todos ellos son temporales y reversibles ${ }^{3}$.

Mostafa ${ }^{15}$ recuerda que es importante no aplicar las inyecciones antes de que su efecto desaparezca por completo para evitar la formación de anticuerpos contra las toxinas, lo que puede llevar a resultados decepcionantes más adelante, por el "efecto vacuna".

Algunas legislaciones que consideran la necesidad permanente de regular, definir criterios y establecer los límites del cirujano dentista en la armonización orofacial describen el veto en la realización de procedimientos invasivos como: alectomía, blefaroplastia, cirugía de elevación de cejas, otoplastia, rinoplastia y ritidoplastia o lifting facial ${ }^{18}$.

\section{Conclusión}

El tratamiento estético dental con ayuda de la toxina botulínica contribuye significativamente a obtener resultados agradables y armoniosos en el tratamiento de la sonrisa gingival, siempre que esté bien indicado, y aunque su efecto sea temporal, se ha convertido en un procedimiento seguro, menos invasivo y eficaz que debe utilizarse siempre que el clínico tenga formación específica y no exceda las dosis recomendadas.

\section{Referencias bibliográficas}

1. Kuhn-Dall'Magro A, Calza SC, Lauxen J, Santos R dos, Valcanaia TC, Dall'Magro. Tratamento do sorriso gengival com toxina botulínica tipo A: relato de caso. RFO UPF 2015;20(1):81-87.

2. Cavalcanti AN, Azevedo JF, Mathias P. Harmonização orofacial: A odontologia além do sorriso. Revista Bahiana de Odontologia. 2017; 8(2):35-36.

3. Matos MB, Valle LSEMB, Ribeiro MA, Naves RC. O uso da toxina botulínica na correção do sorriso gengival revisão de literatura. Braz J Periodontol. 2017;27(3):2936.

4. Sucupira E, Abramovitz A. A simplified method for smile enhancement: botulinum toxin injection for gummy smile. Plast Reconstr Surg. 2012 Sep;130(3):726-728. DOI: 10.1097/PRS.0b013e31825dc32f.

5. Aly LA, Hammouda NI. Botox as an adjunct to lip repositioning for the management of excessive gingi- 
val display in the presence of hypermobility of upper lip and vertical maxillary excess. Dent Res J (Isfahan). 2016 Nov-Dec;13(6):478-483. DOI: 10.4103/17353327.197039.

6. Nasr MW, Jabbour SF, Sidaoui JA, Haber RN, Kechichian EG. Botulinum Toxin for the Treatment of Excessive Gingival Display: A Systematic Review. Aesthet Surg J. 2016 Jan;36(1):82-8. DOI: 10.1093/asj/sjv082.

7. Macedo ACVB de, Nunes VHS, Sardenberg C, Monte Alto R, Almeida RR, Alves Jr J, y col. O sorriso gengival - tratamento basedo na etiologia: uma revisão de literatura. Periodontia 2012;22(4):36-44.

8. Polo M. Botulinum toxin type A in the treatment of excessive gingival display. Am J Orthod Dentofacial Orthop. 2005;127(2):214-8. DOI: 10.1016/j.ajodo.2004.09.013.

9. Mazzuco R, Hexsel D. Gummy smile and botulinum toxin: a new approach based on the gingival exposure area. J Am Acad Dermatol. 2010 Dec;63(6):1042-51. DOI: 10.1016/j.jaad.2010.02.053

10. Moreira DC, Possidônio FS. Aplicação da toxina botulínica tipo A no sorriso gengival: relato de caso. RGO, Rev. Gaúch. Odontol. 2019;67: e20190013. DOI: $10.1590 / 1981-86372019000133555$

11. Chagas TF, Almeida NV de, Lisboa CO, Ferreira DMTP, Mattos CT, Mucha JN. Duration of effectiveness of Botulinum toxin type $\mathrm{A}$ in excessive gingival display: a systematic review and meta-analysis. Braz Oral Res. 2018;32:e30. DOI: 10.1590/1807-3107bor-2018. vol32.0030
12. Pedron IG, Aulestia-Viera PV. La toxina boyulínica como adyuvante em el tratamento de la sonrisa gingival. Rev Clín de Periodoncia Implantol Rehabil Oral. 2017;10(2):87-89. DOI: 10.1016/j.piro.2016.06.001

13. Madeira MC. Anatomia do Dente. 2a ed. São Paulo : Sarvier; 1999.

14. De Paulo EV, Oliveira RCG de, De Freitas KMS. Correção do sorriso gengival com toxina botulínica e outros procedimentos. Revista Uningá. 2018;55(53):1-11.

15. Mostafa D. A successful management of sever gummy smile using gingivectomy and botulinum toxin injection: A case report. Int J Surg Case Rep. 2018;42:169-174. DOI: $10.1016 /$ j.ijscr.2017.11.055

16. Seixas MR, Costa-Pinto RA, Araújo TM de. Checklist dos aspectos estéticos a serem considerados no diagnóstico e tratamento do sorriso gengival. Dental Press J Orthod. $2011 ; 16(2): 131-157$.

17. Silva Neto JMA, Batista ARC, Barros ÍRV, Araujo YBM, Duarte IKF, Tenório Neto JF. Protocolos de Aplicação de Toxina para Sorriso Gengival: uma revisão de literatura. Revista Eletrônica Acervo Saúde. 2019;28:e1079. DOI: 10.25248/reas.e1079.2019.

18. Resolução No CFO-230, [en línea] Brasil: Conselho Federal de Odontologia; 2020 [Consultado en agosto 2020]. Disponible en: http://sistemas.cfo.org.br/visualizar/atos/ RESOLU\%C3\%87\%C3\%83O/SEC/2020/230 